\title{
OPEN A critical evaluation of visual proportion of Gleason 4 and maximum cancer core length quantified by histopathologists
}

Lina Maria Carmona Echeverria ${ }^{1,2 \otimes}$, Aiman Haider ${ }^{3}$, Alex Freeman ${ }^{3}$, Urszula Stopka-Farooqui ${ }^{1}$, Avi Rosenfeld ${ }^{4}$, Benjamin S. Simpson ${ }^{1}$, Yipeng Hu ${ }^{5}$, David Hawkes ${ }^{5}$, Hayley Pye ${ }^{1}$, Susan Heavey ${ }^{1}$, Vasilis Stavrinides ${ }^{1,2}$, Joseph M. Norris ${ }^{1,2}$, Ahmed El-Shater Bosaily ${ }^{2,6}$, Cristina Cardona Barrena ${ }^{1}$, Simon Bott ${ }^{7}$, Louise Brown ${ }^{8}$, Nick Burns-Cox ${ }^{9}$, Tim Dudderidge ${ }^{10}$, Alastair Henderson ${ }^{11}$, Richard Hindley ${ }^{12}$, Richard Kaplan ${ }^{8}$, Alex Kirkham ${ }^{5,13}$, Robert Oldroyd ${ }^{14}$, Maneesh Ghei ${ }^{15}$, Raj Persad ${ }^{16}$, Shonit Punwani ${ }^{5,13}$, Derek Rosario ${ }^{17}$, Iqbal Shergill18, Mathias Winkler ${ }^{19}$, Hashim U. Ahmed ${ }^{19,20}$, Mark Emberton ${ }^{2} \&$ Hayley C. Whitaker ${ }^{1}$

Gleason score 7 prostate cancer with a higher proportion of pattern 4 (G4) has been linked to genomic heterogeneity and poorer patient outcome. The current assessment of G4 proportion uses estimation by a pathologist, with a higher proportion of $\mathrm{G} 4$ more likely to trigger additional imaging and treatment over active surveillance. This estimation method has been shown to have inter-observer variability. Fifteen patients with Prostate Grade Group (GG) 2 (Gleason 3 + 4) and fifteen patients with GG3 (Gleason 4 + 3) disease were selected from the PROMIS study with 192 haematoxylin and eosinstained slides scanned. Two experienced uropathologists assessed the maximum cancer core length (MCCL) and G4 proportion using the current standard method (visual estimation) followed by detailed digital manual annotation of each G4 area and measurement of MCCL (planimetric estimation) using freely available software by the same two experts. We aimed to compare visual estimation of G4 and MCCL to a pathologist-driven digital measurement. We show that the visual and digital MCCL

\footnotetext{
${ }^{1}$ Molecular Diagnostics and Therapeutics Group, Division of Surgery and Interventional Science, University College London, Charles Bell House, 43-45 Foley Street, London W1W 7TS, UK. ${ }^{2}$ Division of Surgery and Interventional Science, Department of Urology, University College London Hospital, 235 Euston Road, London NW1 2BU, UK. ${ }^{3}$ Department of Pathology, University College London Hospital, 60 Whitfield Street, London W1T4EU, UK. ${ }^{4}$ Department of Computer Science, Jerusalem College of Technology, Havaad Haleumi 21, Givat Mordechai, 91160 Jerusalem, Israel. ${ }^{5}$ Centre for Medical Image Computing, University College London, Charles Bell House, 43-45 Foley Street, London W1W 7TS, UK. 'Department of Radiology, Royal Free London NHS Foundation Trust, Pond Street, London NW3 2QG, UK. ${ }^{7}$ Department Urology, Frimley Park Hospital, Frimley Health NHS Foundation Trust, Portsmouth Road, Camberley, Surrey GU16 7UJ, UK. ${ }^{8} \mathrm{MRC}$ Clinical Trials Unit at UCL, 90 High Holborn, London WC1V 6LJ, UK. ${ }^{9}$ Department of Urology, Musgrove Park Hospital, Taunton and Somerset NHS Foundation Trust, Taunton TA1 5DA, UK. ${ }^{10}$ Department of Urology, University Hospital Southampton NHS Foundation Trust, Tremona Road, Southampton, Hampshire SO16 6YD, UK. ${ }^{11}$ Department of Urology, Maidstone and Tunbridge Wells NHS Trust, Hermitage Lane, Tunbridge Wells ME16 900, UK. ${ }^{12}$ Department of Urology, Hampshire Hospitals NHS Foundation Trust, Aldermaston Road, Basingstoke, Hampshire RG24 9NA, UK. ${ }^{13}$ Department of Radiology, UCLH NHS Foundation Trust, University College London Hospital, 235 Euston Road, London NW1 2BU, UK. ${ }^{14}$ Public and Patient Representative, 19 Exbury Gardens, West Bridgford, Nottingham NG2 7SL, UK. ${ }^{15}$ Department of Urology, Whittington Health NHS Trust, Magdala Avenue, London N19 5NF, UK. ${ }^{16}$ Department of Urology, North Bristol NHS Trust, Southmead Road, Westbury-on-Trym, Bristol BS10 5NB, UK. ${ }^{17}$ Department of Urology, Sheffield Teaching Hospitals NHS Foundation Trust, Royal Hallamshire Hospital, Glossop Road, Sheffield, South Yorkshire S10 2JF, UK. ${ }^{18}$ Department of Urology, Wrexham Maelor Hospital NHS Trust, Croesnewydd Road, Wrexham LL13 7TD, UK. ${ }^{19}$ Department of Urology, Imperial College London, South Kensington Campus, London SW7 2AZ, UK. ${ }^{20}$ Imperial Prostate, Division of Surgery, Department of Surgery and Cancer, Faculty of Medicine, Imperial College London, South Kensington Campus, London SW7 2AZ, UK. ${ }^{\square}$ email: l.echeverria@ucl.ac.uk
} 
measurement differs up to $2 \mathrm{~mm}$ in 76.6\% (23/30) with a high degree of agreement between the two measurements; Visual gave a median MCCL of $10 \pm 2.70 \mathrm{~mm}$ (IQR 4, range 5-15 mm) compared to digital of $9.88 \pm 3.09 \mathrm{~mm}$ (IQR 3.82, range 5.01-15.7 $\mathrm{mm})(p=0.64)$ The visual method for assessing G4 proportion over-estimates in all patients, compared to digital measurements [median $11.2 \%$ (IQR 38.75 , range 4.7-17.9\%) vs 30.4\% (IQR 18.37, range 12.9-50.76\%)]. The discordance was higher as the amount of $\mathrm{G} 4$ increased (Bias 18.71, $\mathrm{Cl} 33.87-48.75, \mathrm{r} 0.7, p<0.0001$ ). Further work on assessing actual $\mathrm{G} 4$ burden calibrated to clinical outcomes might lead to the use of differing $\mathrm{G} 4$ thresholds of significance if the visual estimation is used or by incorporating semi-automated methods for G4 burden measurement.

Gleason pattern 4 (G4) prostate cancer is genetically distinct from Gleason pattern 3 and correlates with worse cancer control outcomes either on active surveillance or following active treatment ${ }^{1,2}$. In 2013 Pierorazio et al., retrospectively reviewed 7850 radical prostatectomy specimens to investigate the short-term biochemical outcome using a prognostic based scoring system called the Prostate Grading Group (GG). By separating the Gleason sum 7 group into $3+4$ and $4+3$, the authors found that men with $4+3$ had worse outcome defined as biochemical recurrence-free survival ${ }^{3}$. These findings were further validated and were subsequently endorsed by the 2014 International Society of Urological Pathology Consensus Conference and the World Health Organization $(\mathrm{WHO})^{4-6}$. Additionally, there is some uncertainty about whether $\% \mathrm{G} 4$ in $3+4$ cancers is also relevant to management and outcome $\mathrm{e}^{7-9}$.

This new classification system calls for improved categorisation of the percentage of G4 (\%G4) in Prostate Cancer $(\mathrm{PCa})$ to allow for better risk stratification and inform treatment decisions $\mathrm{s}^{7,9-12}$. The distinction between Gleason $3+4$ (GG2) and $4+3$ (GG3) is made when \%G4 falls below or above $50 \%$, respectively, as visually estimated by a uropathologist ${ }^{5}$. Additionally, the maximum amount of cancer in any core (maximum cancer core length, MCCL) has been used as a proxy for tumour volume estimation and can be used to define clinical significance ${ }^{13,14}$.

Most histological prostate cancer burden studies have been performed in radical prostatectomy specimens or on men who have undergone transrectal systematic biopsies. The Prostate MR Imaging Study (PROMIS) includes men who are biopsy naïve whose prostates were systematically sampled every $5 \mathrm{~mm}$ providing a unique opportunity to perform an in-depth pathologist-driven annotation and digital analysis of the pathological slides and compare this to the visually-reported $\% \mathrm{G} 4$ and $\mathrm{MCCL}^{15}$.

In this study, we aimed to compare \%G4 and MCCL within standard practice, estimated by a pathologist, to a calculated burden from digitally annotated slides by the same pathologists on thirty patients from the PROMIS study with GG2 and GG3 PCa.

\section{Results}

Comparison between visual and digital MCCL. When comparing visual versus digital MCCL, in 23 of the 30 patients the difference was up to $\pm 2 \mathrm{~mm}$; taking into account the positive and negative values the median difference was $0.58 \mathrm{~mm}$ (range -4.12 to $+5.52 \mathrm{~mm}$, t-test, $\mathrm{p}=0.64$ ) (Fig. 1A,B). Seven patients had measurements that differed by $\geq 2 \mathrm{~mm}$ between digital and visual estimation. When viewed as a density plot, there was a tendency to overestimate MCCL in the $3+4$ group and under-estimate in the $4+3$ group when using the visual method (Fig. 1C). To understand the degree of agreement between the two measurements, a Bland-Altman test was performed $^{16}$. There was no systematic difference (bias) between the visual and digital assessment of MCCL, and there was no correlation between increasing MCCL and the level of disagreement between the two measurements (Supplementary figure S.1).

Gleason 4. The visual $\% \mathrm{G} 4$ overestimated $\% \mathrm{G} 4$ burden when compared to the digital assessment in all cases (Fig. 2A). The $4+3$ group had a mean difference of $+26.6 \%$ (range $9.6-41.9 \%$ ) compared to $+10.8 \%$ (range $1.3-24.9 \%$ ) for the $3+4$ group (t-test, $p=1.9 \times 10^{-5}$ ). The average $\% \mathrm{G} 4$ in the patients graded $3+4$ was $11.2 \%$ (range $4.7-17.9 \%$ ) compared to $30.4 \%$ (range 12.9-50.6\%) in the $4+3$ group ( $\mathrm{t}$-test, $p<0.0001$ ). When pathologists were asked to assess the overall Gleason score based on the digital images (visual \%G4), two patients were downgraded from their original clinical grading of $4+3$ to $3+4$ by both pathologists (See yellow bars of patients 23 and 18 in Fig. 2A).

Using the established 50\% G4 threshold to designate a $4+3$ cancer, and based on the digital \%G4 (blue bars), only one patient (number 19 in Fig. 2A) would be classified as $4+3$. When dividing the digital \%G4 into quartiles, two patients in the original $4+3$ group had less $\% \mathrm{G} 4$ than the upper quartile of the $3+4$ group (18 and 30). In other words, these two patients had less \%G4 than the men with the highest \%G4 compromise in the original $3+4$ group. Figure 2B shows the Bland-Altman analysis; showing that there was a bias towards overestimation in the visual estimations as all values are located above the line of complete agreement (Complete agreement would result in a zero value). The disagreement was larger when more than $20 \%$ of G4 was present (R $0.79, p<0.0001)$.

Examination of the index block (block with the highest Gleason score and MCCL), revealed the same findings as previously seen with all tumour containing cores (Fig. 3A). The visual assessment of digitised images downgraded four patients index block from $4+3$ to $3+4$ (patients $18,30,16$, and 23). When examining the digital \%G4, only two patients reached the $50 \%$ G4 threshold (27 and 19), and so would be the only two patients with $4+3$ disease based on digital measurement. The Bland-Altman analysis revealed a similar trend to that of the overall \%G4 analysis. One measurement had a complete agreement between the digital and visual estimate (Patient 6 in Fig. 3A). One patient had a higher digital estimation compared to the visual estimation (Patient 4 in 
A

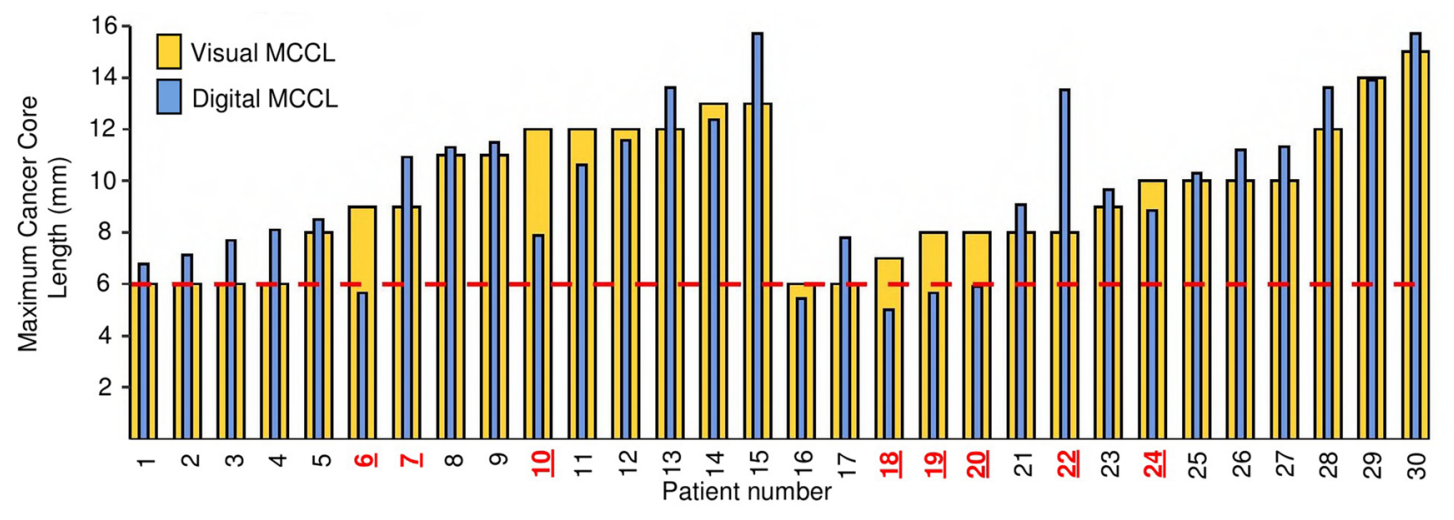

$3+4$

$4+3$

Original glass Gleason score

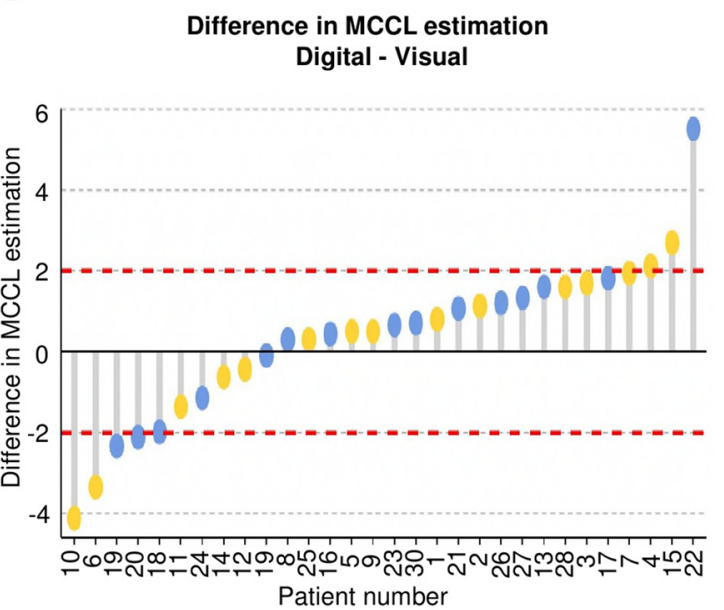

C

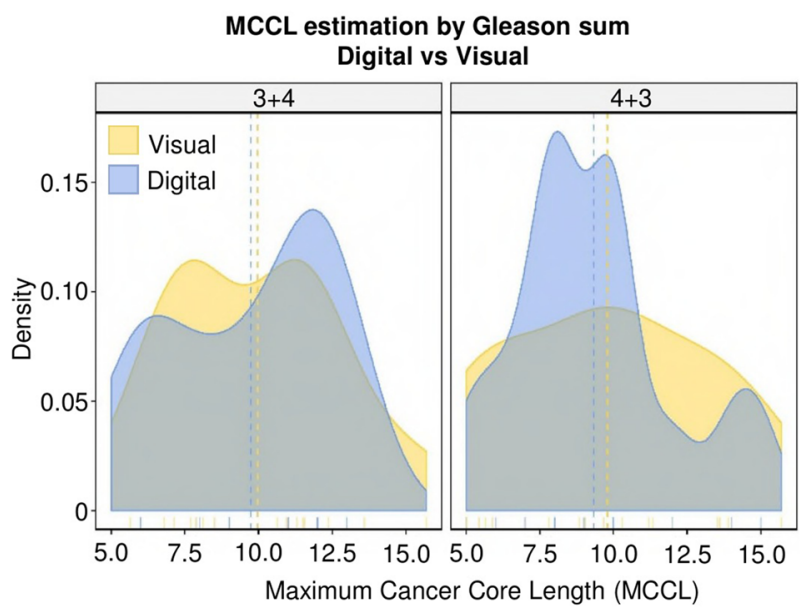

Figure 1. Objective measurement of MCCL and shows a discrepancy with visual measurement and pathologist estimation. (A) MCCL difference between visual and digital MCCL shows under-estimation in visual compared to digital MCCL. Bar plot of visual MCCL in yellow and digital MCCL in blue, organised by Gleason score. MCCL is plotted on the y-axis; each patient is plotted on the $\mathrm{x}$-axis. Red dashed lines represent a threshold of $6 \mathrm{~mm}$ as the MCCL criterion for significance (PROMIS definition 1). Patients highlighted in red were over or underestimated in the original visual measurement. (B) Waterfall plot representing the difference between visual and digital measurements as digital MCCL-visual MCCL by Gleason score (y-axis), patients plotted on the $\mathrm{x}$-axis. Visual Gleason score is represented in yellow for $3+4$ and blue for $4+3$. Bars with a negative value represent measurements where the visual MCCL was shorter than the digital MCCL (underestimation). Bars with a positive value represent cases were the visual MCCL was higher than the digital MCCL. The difference in $80 \%$ of cases is $\pm 2 \mathrm{~mm}(\mathrm{n}=24)$, red dashed line at -2 and $2 \mathrm{~mm}$ difference. (C) Density plots representing the MCCL distribution between visual and digital images by Gleason scores. Y-axis represents the Kernel density estimation. The X-axis contains MCCL values. Visual MCCL score is represented in yellow and blue for the digital measurement. 4+3.The mean visual MCCL was $9.53 \mathrm{~mm}(5-15 \mathrm{~mm})$ and the mean digital MCCL was $9.88 \mathrm{~mm}(5.01-15.74)$.

Fig. 3A). This is represented by the only dot in the negative area of Fig. 3B. The disagreement between measurements increased as the amount of \%G4 increased ( $0.6, p<0.0001)$.

When patients were classified using the clinical significance criteria used in PROMIS in which MCCL and Gleason score were combined to derive definitions $1(\geq 4+3$ or $\geq 6 \mathrm{~mm})$ and $2(\geq 3+4$ or $4 \mathrm{~mm})$ the digital analysis reclassified four patients' index block as lower risk ${ }^{13}$. When all blocks were compared using this system, 20 patients had discrepancy between the visual and digital classification, leading to reclassification to higher or lower risk in six and fourteen patients, respectively (Supplementary Figure S2).

\section{Discussion}

We have presented an in-depth analysis of 30 men from the PROMIS trial, to establish the level of agreement between the gold standard visual estimation of MCCL and \%G4, compared to digitally annotated images. Limitations to this study include: The presence of cribriform pattern was not recorded separately in this study or 
A

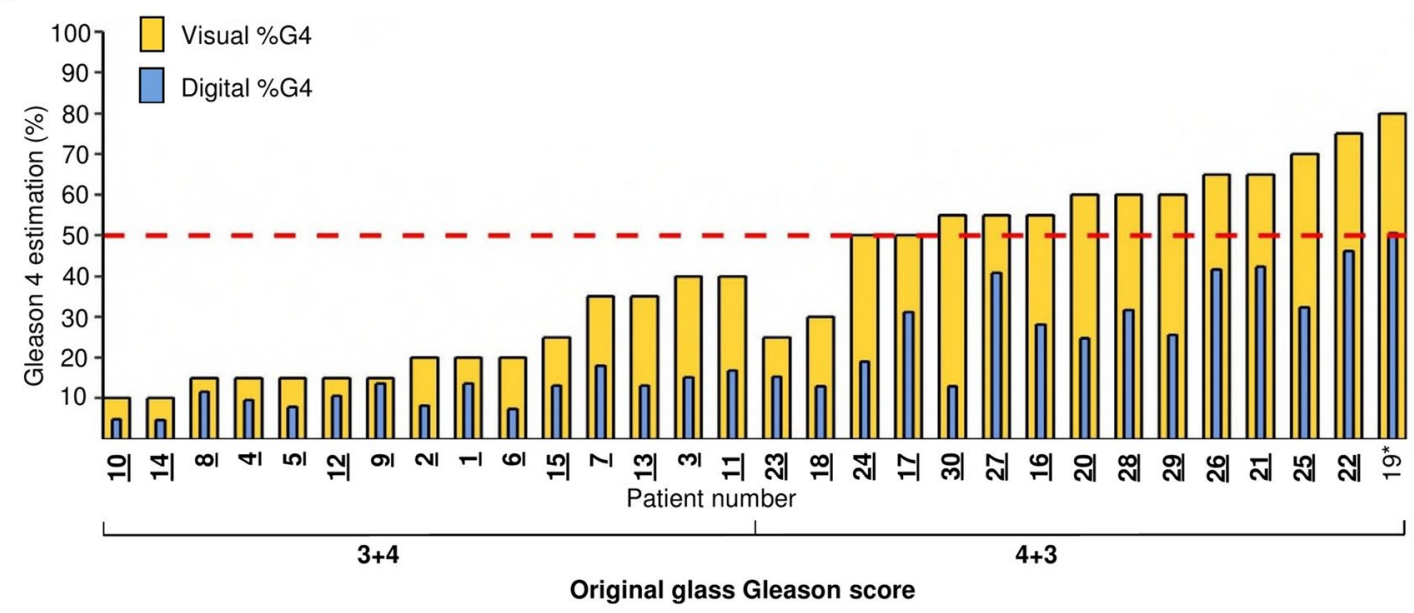

B

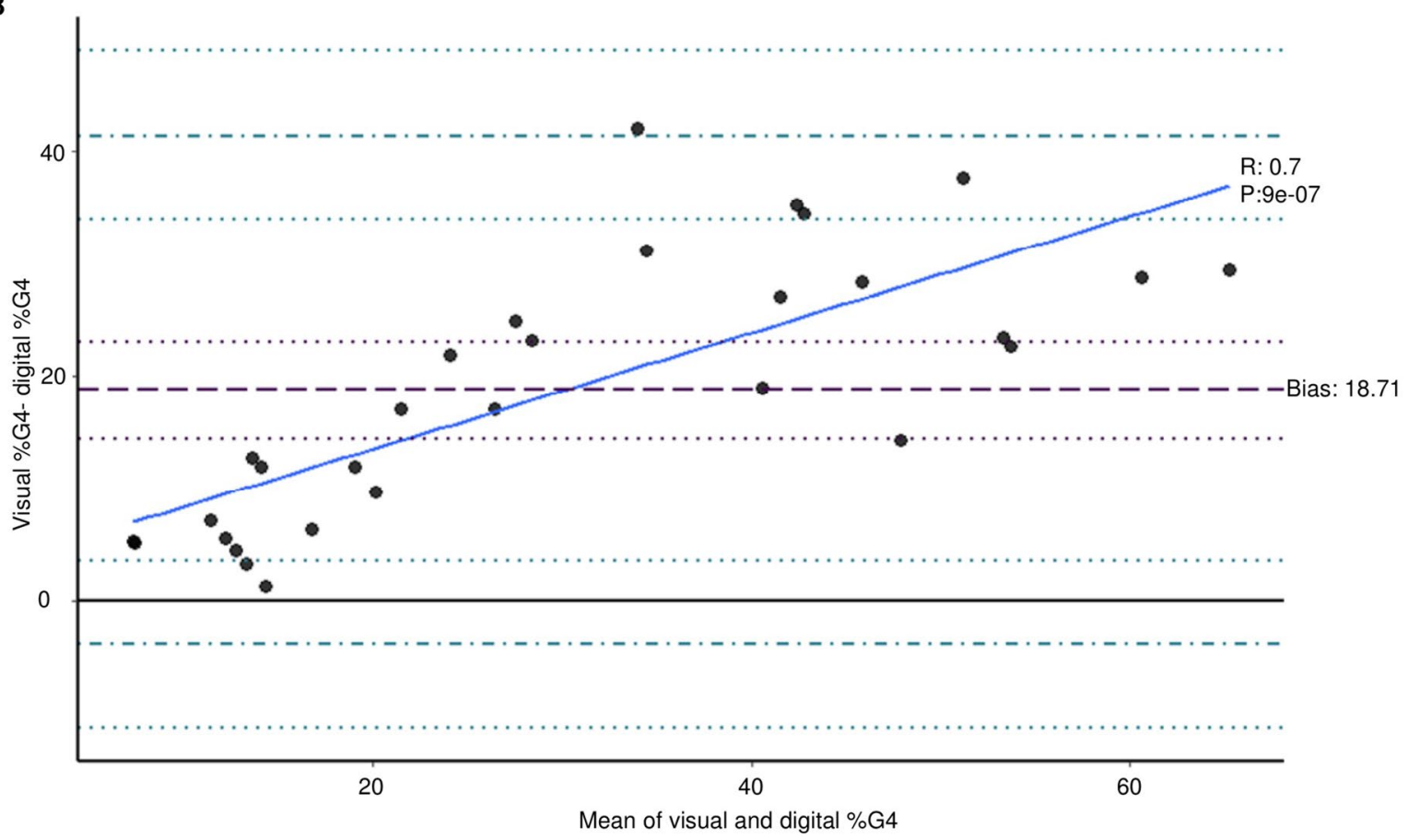

Figure 2. Visual Gleason 4 appraisal overestimates burden of disease. (A) Bar plot of the proportion of Gleason 4 estimation average between two uropathologists (yellow) and digital estimation (blue). \%G4 is plotted on the $\mathrm{y}$-axis; each patient is plotted on the $\mathrm{x}$-axis. A threshold of $50 \% \mathrm{~g} 4$ for clinical significance is shown as a red dashed line. Patient number on the $\mathrm{x}$-axis is highlighted in bold and underlined if the digital measurement of their \%G4 would lead to reclassification based on the digital value. Patient marked with * has $\geq 50 \%$ G4 in the digital measurement. (B) Bland-Altman plot representing the difference in measurement in the $\mathrm{y}$-axis as visual \%G4 - digital \%G4. The $\mathrm{x}$-axis represents the mean $\% \mathrm{G} 4$ measurement of both techniques as (visual \%G4 + digital \%G4)/2. The bold black line represents complete agreement at 0 . The purple dashed line corresponds to the bias at 18.71; the dotted purple line corresponds to the bias confidence interval (33.87-48.75). Dash and dotted blue lines correspond to the upper and lower limit of agreement and confidence intervals are plotted with dotted blue lines. Upper limit of agreement: 41.31 (33.87-48.75), lower limit of agreement: -3.87 ( -11.31 to 3.56 ). Regression line is plotted as a continuous blue line.

included in the final analysis. In addition, the pathologists retrospectively assessed \%G4 on annotated images, introducing potential bias in their assessment. Finally, no long term follow up currently exists for the PROMIS study, so we are unable to determine the prognostic significance of our findings.

A threshold of $4 \mathrm{~mm}$ and $6 \mathrm{~mm}$ has been shown to correlate with $95 \%$ of lesions that have a volume higher than $0.2 \mathrm{~mL}$ or $0.5 \mathrm{~mL}$, respectively ${ }^{13}$. Demetrios et al. found that MCCL greater than $10 \mathrm{~mm}$ can predict T3 disease and large tumour volumes with a hazard ratio (HR) of $5.73^{14}$. Using these thresholds and taking into account the difference in the MCCL measurements, there is a potential impact on the treatment options offered. For instance, patients reclassified as having $<6 \mathrm{~mm}$ MCCL could be candidates for active surveillance instead of radical therapy (Patients 6, 18, 19 and 20) (Fig. 1A,B). Interestingly, the visual measurement of men with $3+4$ 
A

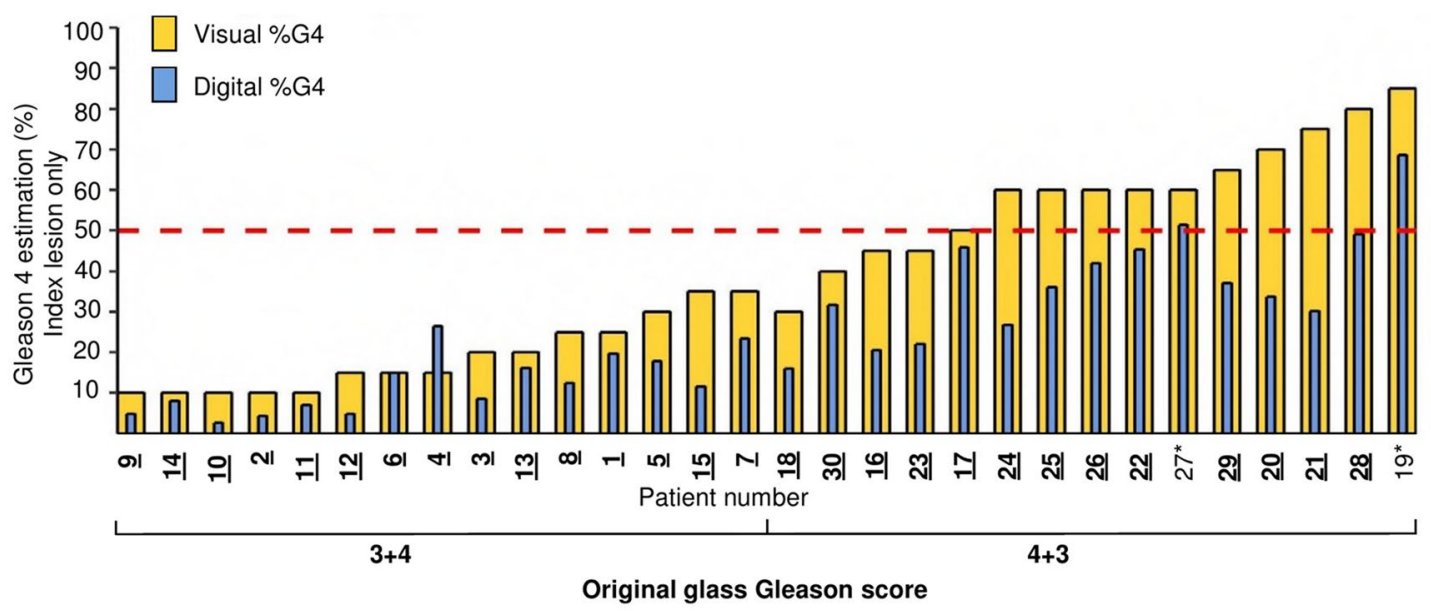

B

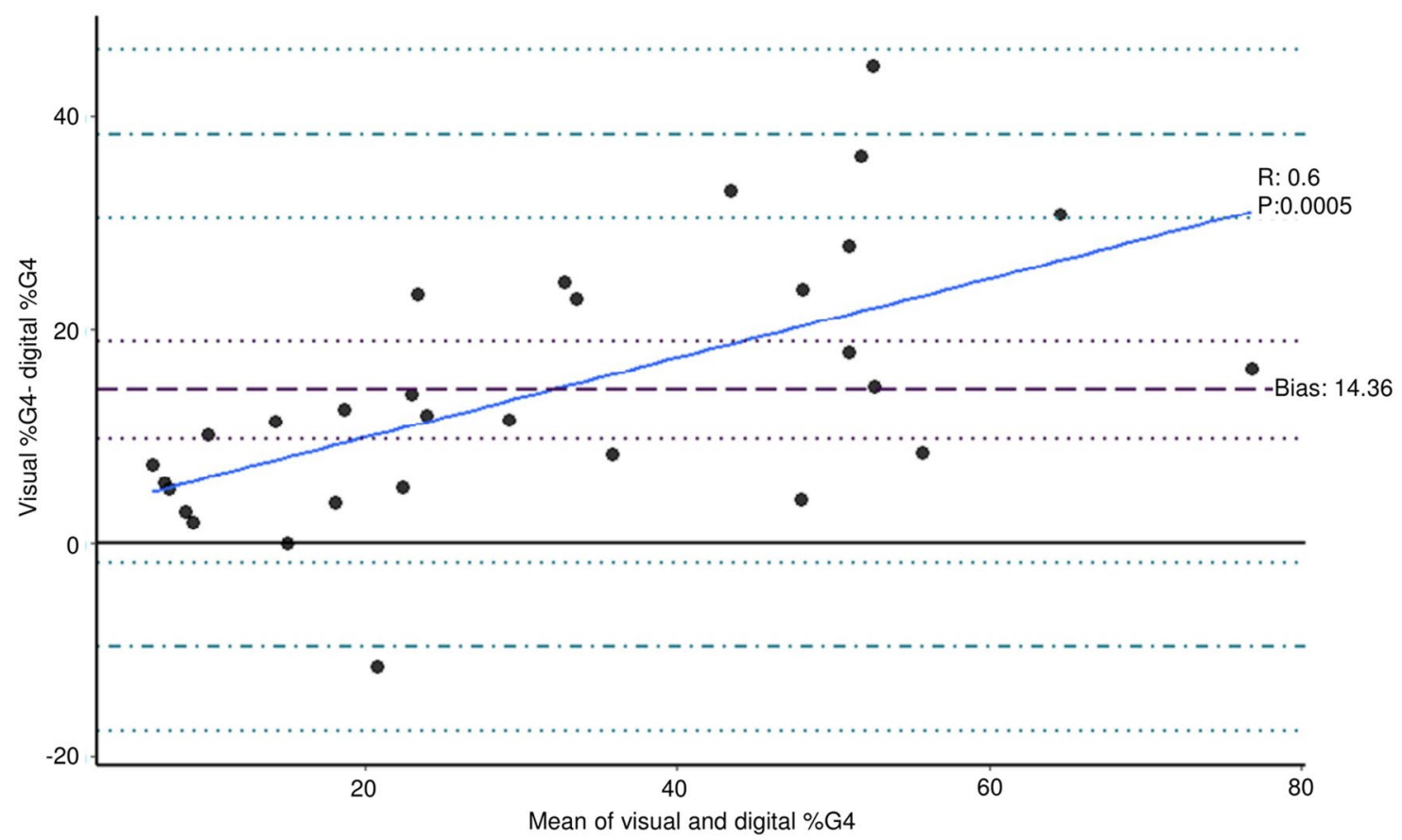

Figure 3. Objective measurement of Gleason 4 burden shows a discrepancy between visual measurement and the digital measurement for the index block. (A) Visual \%G4 for the index block 30 patients shown in yellow overlaid with digital \%G4 in blue. Patients separated by original Gleason grade grouping; $3+4$ or $4+3$, and organized by visual $\% \mathrm{G} 4$. A threshold of $50 \% \mathrm{G} 4$ for clinical significance is shown as a red dashed line. Patient number on the $\mathrm{x}$-axis highlighted in bold and underlined if the objective measurement of their $\% \mathrm{G} 4$ would cause reclassification. (B) Bland-Altman plot representing the difference in measurement in the $\mathrm{y}$-axis as visual \%G4 - digital \%G4. The $\mathrm{x}$-axis represents the mean $\% \mathrm{G} 4$ measurement of both techniques as (visual \%G4 + digital \%G4)/2. The bold black line represents complete agreement at 0 . The purple dashed line corresponds to the bias at 14.36; the dotted purple line corresponds to the bias confidence interval (9.78-18.94). Dash and dotted blue lines correspond to the upper and lower limit of agreement and confidence intervals are plotted with dotted blue lines. Upper limit of agreement: 38.40 (30.49-46.32), lower limit of agreement: -9.67 $(-17.59$ to -1.76$)$. The regression line is plotted as a continuous blue line.

disease was more likely to be greater compared to men with $4+3$ disease (Fig. 1C). Despite these differences, the Bland-Altman analysis showed good concordance between the two measurements; thus, the accuracy of the MCCL is not compromised when a digital tool is used.

In our study, the visual estimation of \%G4 differed from the digital one; accurate measurement of the G4 burden has been shown to help risk-stratify patients ${ }^{9,17}$. In a study by de Souza et al., $20 \%$ of Gleason $3+4$ tumours had more extensive G4 disease than the first quartile of $4+3$ tumours in radical prostatectomy specimens ${ }^{18}$. In 2014 , Huang et al. found that $45 \%$ of men with $\leq 5 \%$ of G4 in prostate biopsy had insignificant cancer in radical prostatectomy ${ }^{7}$. Additionally, several papers have shown that tumours with lower \%G4 behave closer to GG1 tumours $3,8,9,19-22$. 


\begin{tabular}{|c|c|c|c|c|c|c|}
\hline \multirow{3}{*}{ Gleason score } & \multicolumn{2}{|c|}{$\begin{array}{l}\text { UCH-PROMIS cohort }(4+3 \\
\text { or } \geq 6 \mathrm{~mm} \text { MCCL })\end{array}$} & \multirow[t]{2}{*}{$\begin{array}{l}p \text { value }(3+4 \text { vs } \\
4+3)\end{array}$} & \multicolumn{2}{|c|}{ Selected 30 patients } & \multirow[t]{2}{*}{$\begin{array}{l}p \text { value }(3+4 \text { vs } \\
4+3)\end{array}$} \\
\hline & $3+4$ & $4+3$ & & $3+4$ & $4+3$ & \\
\hline & $\mathrm{n}=67(78 \%)$ & $\mathrm{n}=18(22 \%)$ & & $\mathrm{n}=15(50 \%)$ & $\mathrm{n}=15(50 \%)$ & \\
\hline Age (years) & $63(43-77)$ & $64(48-79)$ & $0.44^{*}$ & $62(50-72)$ & $65(48-79)$ & $0.30^{*}$ \\
\hline $\begin{array}{l}\text { Prostate volume } \\
(\mathrm{cc})\end{array}$ & $38.34(16-83)$ & $38.18(26-55)$ & $0.65^{\star *}$ & $34(21-62)$ & $38(26-55)$ & $0.11^{\star *}$ \\
\hline $\begin{array}{l}\text { Presenting PSA } \\
(\mathrm{ng} / \mathrm{dL})\end{array}$ & $7.46(1.30-13)$ & $10.76(5.7-15)$ & $<0.0001^{*}$ & $7.60(4.9-10.1)$ & $10.74(6.2-15)$ & $0.0005^{\star}$ \\
\hline $\begin{array}{l}\text { PSA density } \\
\text { (PSAd) }\end{array}$ & $0.22(0.06-0.59)$ & $0.29(0.11-0.53)$ & $0.002^{* *}$ & $0.24(0.10-0.38)$ & $0.29(0.11-0.53)$ & $0.14^{*}$ \\
\hline Likert 2 & $1(1.4 \%)$ & 0 & & 0 & 0 & \\
\hline Likert 3 & $8(11.9 \%)$ & $3(16.6 \%)$ & & $1(6.6 \%)$ & 0 & \\
\hline Likert 4 & $21(31.3 \%)$ & $3(16.6 \%)$ & & $6(40 \%)$ & $4(26.6 \%)$ & \\
\hline Likert 5 & $5(7.46 \%)$ & $12(66.6 \%)$ & & $8(53.3 \%)$ & $11(73.3 \%)$ & \\
\hline Likert NA & $4(5.87 \%)$ & 0 & & 0 & $1(6.6 \%)$ & \\
\hline
\end{tabular}

Table 1. Gleason 7 patients in the PROMIS cohort and 30 selected patients for in-depth analysis. Table comparing the Gleason 7 patients from University College London (UCH) within the PROMIS study. UCH PROMIS cohort is on the left, selected patients on the right. Number of patients per group by Gleason score in each cohort as $n=$, percentage in parenthesis. Mean value for age, prostate volume, presenting PSA and PSA density, with range in parenthesis. Age is denoted in years, prostate volume in cubic centimetres (cc), PSA in $\mathrm{ng} / \mathrm{dL}$ and PSA density calculated as PSA/prostate volume. Likert scores are presented as number of patients and percentage in parenthesis, Likert NA when no Likert score was given. ${ }^{\star} p$-value obtained using an unpaired t-test, ${ }^{* *}$ if using Mann-Whitney test.

In this study, we found that visual estimation always overestimated the amount of G4 compared to a digitally calculated \%G4. For all of these patients, reclassification of the \%G4 would potentially lead to a change in treatment options, and imaging follow up. For example, patient 18 was reclassified after digital assessment and would be downgraded from $4+3$ of $>6 \mathrm{~mm}$ to $3+4$ of $<6 \mathrm{~mm}$ (Fig. 2A). The same was found when we examined the index block only.

Integration of \%G4 reporting in biopsies and radical prostatectomy specimens is already recommended ${ }^{6}$. The findings of our study suggest that a re-assessment of \%G4 estimation may be required. Reclassification of G4 could lead to a re-evaluation of previously published biomarker and clinical studies and redefine the reference standard for research. The heterogeneity of studies of the prognostic importance of Gleason $3+4$ disease as compared with Gleason $4+3$ disease may be a reflection of uncertainty about how much G4 pattern disease is actually shown in specimens and is particularly relevant to treatments such as radiotherapy or ablation where there is no whole mount radical prostatectomy specimen to analyse.

As we move toward the inclusion of digital pathology in standard clinical practice, it will be essential to investigate the differences between human and digital estimation of key pathological parameters and the potential impact this could have on patient care. This will involve adapting the current visual classification to digitallyderived grading. This study does not aim to highlight human error or criticize visual estimation of the pathologists but to encourage the use of technology to improve our understanding of MCCL and G4 burden in prostate cancer, and to seek novel methods to quantify and study the disease. Whilst this type of analysis would be currently challenging to embed directly into clinical practice due to the time taken to contour each region; work is already ongoing to automate this process ${ }^{23-28}$. Identifying relatively overlooked elements, such as $\% \mathrm{G} 4$, improves the accuracy of the models used in machine learning ${ }^{29}$, as such future algorithms can be trained to specifically identify $\% \mathrm{G} 4$, rather than GG alone.

Further research is also needed to develop and validate new thresholds of the burden of G4 against large cohorts with medium and long-term cancer control outcomes.

\section{Materials and methods}

Patients. Two-hundred and twenty-six patients from University College London Hospital took part in the PROMIS trial. Men underwent $5 \mathrm{~mm}$ sampling using a transperineal template mapping procedure. Of 113 men with Gleason $7 \mathrm{PCa}, 85$ had significant disease (PROMIS definition 1: Gleason $4+3$ or MCCL $\geq 6 \mathrm{~mm}$ ). 15 patients with Gleason $3+4$ and 15 patients with $4+3$ disease were selected from the 85 , using a random number generator (Table 1; Fig. 4A). A mean of $14.2 \pm 8.05$ cores per patient (IQR 9, range 2-34) were taken. $192 \mathrm{H} \& \mathrm{E}$ slides from these 30 patients were scanned using a NanoZoomer-SQ digital slide scanner (Hamamatsu).

Digital scan annotations and data collection. Two experienced UCH uropathologists with 16 years $(\mathrm{AF})$ and 1.5 years' experience $(\mathrm{AH})$ were involved in this study. The 30 cases included in this study were originally reported by AF as part of the PROMIS trial. The pathologists were blinded to the PROMIS Gleason score; scans were shown randomly and assessed by two experienced uropathologists (AF/AH) using NDP.View 2 software. Each slide was systematically assessed as follows: 1 . Each core was numbered from left to right. 2 . Length 
A

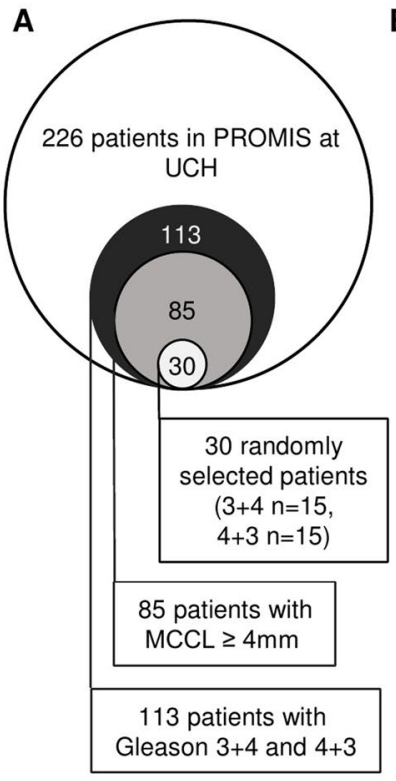

B

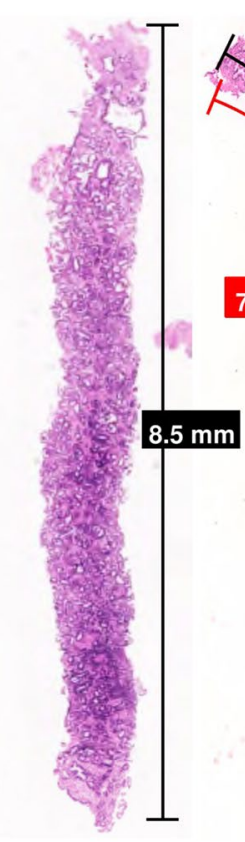

C

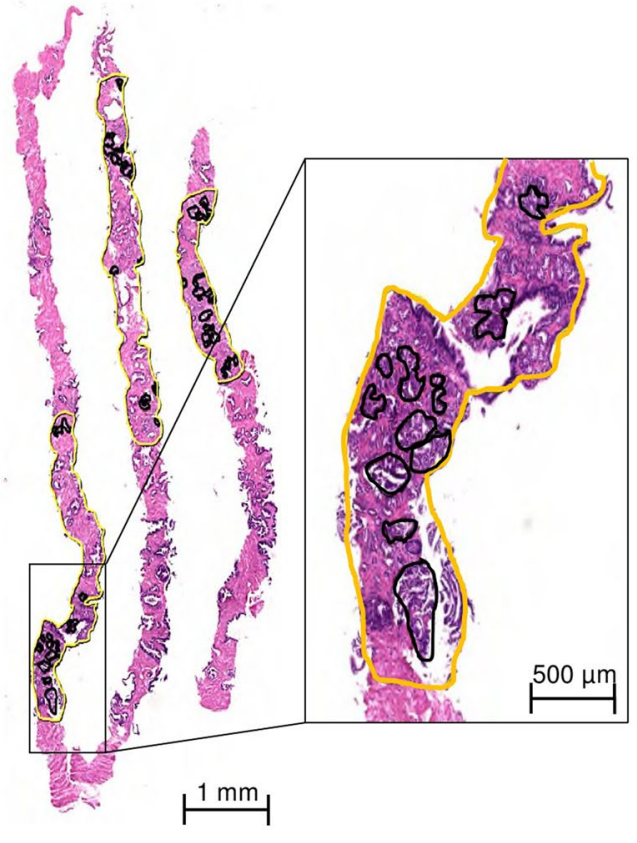

D

$$
\text { Equation 1: } \quad \text { Percentage G4 }=\frac{\text { Total area contoured in black }}{\text { Total area contoured in yellow }} \times 100
$$

Figure 4. Patient selection and methods of digital manual annotation. (A) Euler diagram representing patient selection process for 30 patients for in-depth analysis. (B) NDPview2 image of scanned H\&E slide of prostate cores from transperineal biopsies, where nuclei are shown in blue, and other structures in pink. From left to right, MCCL measurement in a straight core of $8.5 \mathrm{~mm}$. Approximate visual pathologist measurement marked with a red line $(7.76 \mathrm{~mm})$. Following the axis of the core, three measurements in black of $2.53 \mathrm{~mm}, 2.11 \mathrm{~mm}$ and $4.48 \mathrm{~mm}$ for a total of $9.12 \mathrm{~mm}$ for the digital measurement. (C) Three prostate cores, areas with cancer were contoured in yellow, areas with Gleason 4 were contoured in black. Close up of contours shown in black box. Non-contoured areas correspond to benign prostatic tissue. (D) Equation used to derive percentage Gleason 4.

of cancer was measured (Fig. 4B). 3. Areas containing any cancer were contoured in yellow (Fig. 4C). 4. Areas containing G4 were contoured in black (Fig. 4C).

The MCCL was reported prospectively by the pathologists during the trial using the integrated ruler in the microscope; this measurement was assigned as 'visual' MCCL. In PROMIS, the MCCL was reported by taking into account intervening benign glands (ISUP) and measuring cancer only. For the purposes of this study, the ISUP measurement was used. The 'digital' MCCL was derived as follows: If a core was straight, a single measurement was performed. If there was any curvature, manual sequential measurements were performed along the core axes and combined to give the final measurement.

$\% \mathrm{G} 4$ was not collected as part of the original trial, pathologists retrospectively visually estimated the \%G4 per patient to the closest $10 \%$ using the annotated images. This was assigned as 'visual' \%G4. For digital \%G4, the software performs instant area measurements. The resulting area (for each yellow and black contours) was prospectively recorded, and an objective percentage of G4 was calculated as shown in equation 1 (Fig. 4D). This total was assigned as 'digital' \%G4. A separate analysis of the index block was performed separately. The index block was defined as the block with the highest Gleason score and MCCL in combination with concordance with the index lesion on mpMRI.

Statistical analysis. Patients were divided according to the original Gleason score from the PROMIS trial into $3+4$ and $4+3$. The routinely performed 'visual' estimation for both measurements was used as the reference standard for all comparisons. When comparing two groups, meeting normal distribution (Shapiro-Wilk test) and same variances (F-test), a student t-test was applied. Whenever data was not normally distributed a MannWhitney test was performed. To quantify the agreement between the two methods, the Bland Altman method was performed. The visual method was used as a standard for comparison; bias was defined as the average of the difference between the two methods. Limits of agreement were calculated at $95 \%$ CI. All analyses were made using R: A Language and Environment for Statistical Computing ${ }^{30}$. The Bland-Altman analysis was performed using the blandr package for $\mathrm{R}^{31}$.

Ethical approval. All clinical samples were collected from University College London Hospital NHS Trust patients who had provided informed consent. Ethics committee approval was granted by National Research 
Ethics Service Committee London (reference 11/LO/0185). Access to biobank samples was obtained [reference $(\mathrm{EC} / 21.16)]$. All analyses were performed in accordance with relevant guidelines and regulations.

Received: 30 April 2020; Accepted: 28 August 2020

Published online: 14 October 2020

\section{References}

1. Rubin, M. A., Girelli, G. \& Demichelis, F. Genomic correlates to the newly proposed grading prognostic groups for prostate cancer. Eur. Urol. 69, 557-560 (2016).

2. Sowalsky, A. G. et al. Gleason score 7 prostate cancers emerge through branched evolution of clonal Gleason pattern 3 and 4. Clin. Cancer Res. 23, 3823-3833 (2017).

3. Pierorazio, P. M., Walsh, P. C., Partin, A. W., Epstein, J. I. \& Epstein, J. Prognostic Gleason grade grouping: data based on the modified Gleason scoring system. BJU Int. 111, 753-760 (2013).

4. Epstein, J. I. et al. A contemporary prostate cancer grading system: a validated alternative to the Gleason score. Eur. Urol. 69, 428-435 (2016).

5. Epstein, J. I. et al. The 2014 International Society of Urological Pathology (ISUP) consensus conference on Gleason grading of prostatic carcinoma definition of grading patterns and proposal for a new grading system. Am. J. Surg. Pathol. 40, 244-252 (2016).

6. Humphrey, P. A., Moch, H., Cubilla, A. L., Ulbright, T. M. \& Reuter, V. E. The 2016 WHO classification of tumours of the urinary system and male genital organs-Part B: prostate and bladder tumours. Eur. Urol. 70, 106-119 (2016).

7. Huang, C. C. et al. Gleason score $3+4=7$ prostate cancer with minimal quantity of Gleason pattern 4 on needle biopsy is associated with low-risk tumor in radical prostatectomy specimen. Am. J. Surg. Pathol. 38, 1096-1101 (2014).

8. Sato, S. et al. Cases having a Gleason Score $3+4=7$ with $<5 \%$ of Gleason pattern 4 in prostate needle biopsy show similar failurefree survival and adverse pathology prevalence to Gleason Score 6 cases in a radical prostatectomy cohort. Am. J. Surg. Pathol. 43, $1560-1565$ (2019).

9. Sauter, G. et al. Clinical utility of quantitative Gleason grading in prostate biopsies and prostatectomy specimens. Eur. Urol. 69, 592-598 (2016).

10. Cole, A. I. et al. Prognostic value of percent Gleason grade 4 at prostate biopsy in predicting prostatectomy pathology and recurrence. J. Urol. 196, 405-411 (2016).

11. Stark, J. R. et al. Gleason score and lethal prostate cancer: does $3+4=4+3$ ?. J. Clin. Oncol. 27, 3459-3464 (2009).

12. Berney, D. M. et al. The percentage of high grade disease in prostate biopsies significantly improves on grade groups in prediction of prostate cancer death. Histopathology 75(4), 589-597 (2019).

13. Ahmed, H. U. et al. Characterizing clinically significant prostate cancer using template prostate mapping biopsy. J. Urol. 186, 458-464 (2011).

14. Simopoulos, D. N. et al. Cancer core length from targeted biopsy: an index of prostate cancer volume and pathological stage. BJU Int. https://doi.org/10.1111/bju.14691 (2019).

15. Ahmed, H. U. et al. Diagnostic accuracy of multi-parametric MRI and TRUS biopsy in prostate cancer (PROMIS): a paired validating confirmatory study. Lancet 389, 815-822 (2017).

16. Bland, J. M. \& Altman, D. G. Applying the right statistics: analyses of measurement studies. Ultrasound. Obstet. Gynecol. 22, 85-93 (2003).

17. Sharma, M. \& Miyamoto, H. Percent Gleason pattern 4 in stratifying the prognosis of patients with intermediate-risk prostate cancer. Transl. Androl. Urol. 7, S484-S489 (2018).

18. de Souza, M. F., de Azevedo Araujo, A. L. C., da Silva, M. T. \& Athanazio, D. A. The Gleason pattern 4 in radical prostatectomy specimens in current practice-quantification, morphology and concordance with biopsy. Ann. Diagn. Pathol. 34, 13-17 (2018).

19. Berg, K. D., Roder, M. A., Brasso, K., Vainer, B. \& Iversen, P. Primary Gleason pattern in biopsy Gleason score 7 is predictive of adverse histopathological features and biochemical failure following radical prostatectomy. Scand. J. Urol. 48, 168-176 (2014).

20. Helpap, B. et al. The significance of accurate determination of Gleason score for therapeutic options and prognosis of prostate cancer. Pathol. Oncol. Res. 22, 349-356 (2016).

21. Miyake, H. et al. Prognostic significance of primary Gleason pattern in Japanese men with Gleason score 7 prostate cancer treated with radical prostatectomy. Urol. Oncol. Semin. Orig. Invest. 31, 1511-1516 (2013).

22. Khoddami, S. M. et al. Predictive value of primary Gleason pattern 4 in patients with Gleason score 7 tumours treated with radical prostatectomy. BJU Int. 94, 42-46 (2004).

23. Esteva, A. et al. A guide to deep learning in healthcare. Nat. Med. 25(1), 24-29 (2019).

24. Ström, P. et al. Artificial intelligence for diagnosis and grading of prostate cancer in biopsies: a population-based, diagnostic study. Lancet Oncol. https://doi.org/10.1016/S1470-2045(19)30738-7 (2020).

25. Nir, G. et al. Automatic grading of prostate cancer in digitized histopathology images: learning from multiple experts. Med. Image Anal. 50, 167-180 (2018).

26. Campanella, G. et al. Clinical-grade computational pathology using weakly supervised deep learning on whole slide images. Nat. Med. 25, 1301-1309 (2019).

27. Arvaniti, E. et al. Automated Gleason grading of prostate cancer tissue microarrays via deep learning. Sci. Rep. 8, 12054 (2018).

28. Nagpal, K. et al. Development and validation of a deep learning algorithm for improving Gleason scoring of prostate cancer. $n p j$ Digit. Med. 2, 1-10 (2019).

29. Rosenfeld, A., Graham, D. G., Hamoudi, R., Butawan, R., Eneh, V., Khan, S. et al. MIAT: a novel attribute selection approach to better predict upper gastrointestinal cancer. in Proceedings of the 2015 IEEE International Conference on Data Science and Advanced Analytics, DSAA 2015 (Institute of Electrical and Electronics Engineers Inc., 2015). https://doi.org/10.1109/DSAA.2015.7344866.

30. Team RC. R: A Language and Environment for Statistical Computing (2019). https://www.r-project.org/.

31. Datta, D. blandr: A Bland-Altman Method Comparison Package for R (2017). https://doi.org/10.5281/zenodo.824514.

\section{Author contributions}

L.M.C.E., H.C.W., H.U.A., and M.E. conceived and designed the study. L.M.C.E., U.S., A.H., A.F., C.C.B. collected the data L.M.C.E. analysed the data with guidance from Y.H., A.R. and L.B. All authors were involved in writing the paper and had final approval of the submitted and published versions.

\section{Competing interests}

Ahmed currently receives funding from the Wellcome Trust, Prostate Cancer UK, Medical Research Council (UK), Cancer Research UK, The Urology Foundation, BMA Foundation, Imperial Healthcare Charity, Sonacare 
Inc., Trod Medical and Sophiris Biocorp for trials in prostate cancer. Ahmed is a paid medical consultant for Sophiris Biocorp, Sonacare Inc., BTG and Boston for trials work and proctoring. Emberton receives funding from NIHR-i4i, MRC, Cancer Research UK, Sonacare Inc., and Sophiris Biocorp for trials in prostate cancer. Emberton is a medical consultant to Sonacare Inc., Sophiris Biocorp, Steba Biotech, Exact Imaging and Profound Medical. Ahmed and Emberton are proctors for HIFU and paid for training other surgeons in this procedure. Ahmed is a proctor for cryotherapy using the Galil/BTG system. Emberton is a proctor for Irreversible Electroporation (Nanoknife). Rest of the authors have no conflict of interest.

\section{Additional information}

Supplementary information is available for this paper at https://doi.org/10.1038/s41598-020-73524-z.

Correspondence and requests for materials should be addressed to L.M.C.E.

Reprints and permissions information is available at www.nature.com/reprints.

Publisher's note Springer Nature remains neutral with regard to jurisdictional claims in published maps and institutional affiliations.

(c) (i) Open Access This article is licensed under a Creative Commons Attribution 4.0 International License, which permits use, sharing, adaptation, distribution and reproduction in any medium or format, as long as you give appropriate credit to the original author(s) and the source, provide a link to the Creative Commons licence, and indicate if changes were made. The images or other third party material in this article are included in the article's Creative Commons licence, unless indicated otherwise in a credit line to the material. If material is not included in the article's Creative Commons licence and your intended use is not permitted by statutory regulation or exceeds the permitted use, you will need to obtain permission directly from the copyright holder. To view a copy of this licence, visit http://creativecommons.org/licenses/by/4.0/.

(C) The Author(s) 2020 